\title{
DIFERENTES TIPOS DE OBJETOS PARA DAR SUPORTE A APRENDIZAGEM
}

\author{
Maria Lucia Pozzatti Flôres

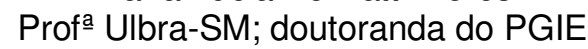 \\ mlflores@terra.com.br \\ Liane Margarida Rockenbach Tarouco \\ Prof. Dr. do PGIE/UFRGS
}

\section{RESUMO}

Neste artigo será mostrado que os tipos diferentes de objeto de aprendizagem contribuem para facilitar o processo de aprendizagem do estudante. Esses objetos podem ser compostos apenas de um elemento ou de vários recursos digitais combinados. Podem ter a lógica e a estrutura para gerar outros objetos, mas acima de tudo devem interagir com os alunos colaborando em sua aprendizagem.

Palavras-Chave: objeto de aprendizagem. Tipos de objetos.

\section{DIFFERENT TYPES OF OBJECTS TO SUPPORT THE LEARNING}

\section{ABSTRACT}

This article will show that different types of learning object help to facilitate the learning process of students. These objects can be composed of only one element or more digital resources combined. They may have the logic and structure to generate other objects, but above all it must interact with students working in their learning.

Keywords: learning object. Types of object.

\section{1-INTRODUÇÃO}

A emergência da Internet e da aprendizagem através de recursos computacionais como meios viáveis de compartilhar e reusar os materiais da instrução, mudaram a visão da educação e a maneira como pode ser deliberada a aprendizagem. Uma quantidade enorme de materiais estão sendo desenvolvidos para suportar a educação e aprendizagem, entretanto avaliar suas qualidade e aplicabilidade para aprendizagem é muito difícil.

Um tipo de material computacional que está sendo muito usado para suportar a aprendizagem é o objeto de aprendizagem. Wiley (2000) entende que os objetos de aprendizagem são elementos de um tipo novo de instrução por computador baseado no paradigma "orientado ao objeto" da informática. Eles são criados por componentes (objetos) que podem ser reusados em diferentes contextos (Dahl \& Nygaard, 1966). Adicionalmente, os objetos de aprendizagem são entidades digitais distribuídas pela Internet, isto significa que todos podem acessá-los e usá-los simultaneamente, ao contrário dos tradicionais meios instrutivos, tal como um vídeo existente em apenas num 
2 lugar. Além disso, as pessoas que usam objetos de aprendizagem podem colaborar sobre ele e beneficiar-se imediatamente das versões novas.

Os princípios para a construção dos objetos de aprendizagem buscam integrar a usabilidade do design e a usabilidade pedagógica. Segundo Martins (2004) a usabilidade de design engloba estudos na área da ergonomia, focados em interface usuário-sistema, conceito que busca definir as características da utilização e do desempenho na interação e leitura das interfaces computacionais pelo usuário. Já a usabilidade pedagógica se refere à necessidade de aprendizagem significativa e à utilização de ambientes para aprendizagem construtivista.

A aprendizagem significativa e o uso da tecnologia estão interrelacionadas e são interativas e interdependentes.

Wiley (2000) desenvolveu uma teoria instrucional que forneceu a orientação no uso instrutivo de objetos de aprendizagem. Este artigo mostra que Wiley ao desenvolver sua teoria instrucional preocupou-se em conciliar cada informação (habilidade) com um tipo de objeto de aprendizagem específico que facilite a aprendizagem. Além disso, esses objetos de aprendizagem devem ter a característica de serem reusados em diferentes contextos.

\section{2- IDÉIAS DE ALGUNS TEÓRICOS SOBRE A APRENDIZAGEM}

Os tecnólogos instrutivos e os psicólogos educacionais têm se empenhado na criação de estratégias de ensino, pois existem poucos métodos formalizados para a criação da teoria instrutiva de um objeto de aprendizagem.

Primeiro, pode-se entender o processo da aprendizagem através de Bruner (1966) que afirmou que a aprendizagem é um processo ativo, no qual os sujeitos constroem novas idéias, ou conceitos, com base nos seus conhecimentos passados e atuais. O aluno seleciona e transforma a informação, constrói hipóteses e toma decisões, utilizando, para isto, a sua estrutura cognitiva. É a estrutura cognitiva (esquemas, modelos mentais) que fornece significado e organização para as experiências e permite ao indivíduo "ir além da informação dada".

Para este autor, o professor deve incentivar os alunos a descobrir por si os princípios e para isso terá de procurar envolver-se com o educando num diálogo ativo. A tarefa do professor é reconstruir a informação a ser aprendida num formato adequado às características e competências atuais do aluno.

Bruner (1966) afirmou que a teoria do ensino deve ser direcionada para quatro aspectos principais: o processo de aprendizagem; modos de estruturação e organização dos conhecimentos (para que sejam facilmente compreendidos pelo aluno); sequências mais efetivas de apresentação dos conteúdos; natureza e ritmo das recompensas e punições.

Um dos mais notáveis experts sobre o paradigma de design instrucional foi Gagné. Gagné (1980) constatou a existência de diferentes tipos ou níveis de aprendizagem e a necessidade de considerar para cada um deles tipos diferentes de instrução. Ele identificou cinco categorias principais na 
3 aprendizagem: informação verbal; competências intelectuais; estratégias cognitivas; competências motoras e atitudes.

Para cada tipo de aprendizagem são necessárias determinadas condições internas e externas. Por exemplo, para que se aprendam estratégias cognitivas, é preciso que existam oportunidades para que se pratique o desenvolvimento de novas soluções para os problemas. Para aprender atitudes, o aluno deve ser colocado em situações em que desempenhe papéis adequados e verossímeis.

Gagné (1980) sugeriu que a aprendizagem de competências intelectuais pode ser organizada numa forma hierárquica de crescente complexidade: reconhecimento de estímulo, geração de resposta, seguir procedimentos, uso da terminologia, discriminações, formação de conceito, aplicação de regras e resolução de problemas. A importância desta hierarquia consiste em permitir identificar os pré-requisitos que devem ser satisfeitos em cada um dos níveis de forma a facilitar a aprendizagem e fornecer uma base para a sequência de instrução.

Merril (1983) desenvolveu um estudo sobre as teorias de design educativo procurando encontrar nelas princípios comuns. Merril definiu os conceitos de "princípio", "prática" e "programa" e analisou as relações que as várias teorias e modelos estabelecem entre eles.

Para este autor, uma teoria deve especificar os princípios e as práticas para os implementar. Deve haver um pequeno conjunto de princípios que suportam todos os programas e práticas (teorias, modelos e métodos).

Merril (1983) assumiu que se um princípio é incluído em várias teorias de ensino, isso significa que ele fundamenta a sua validade na experiência ou investigação. Desta forma os princípios enunciados, na medida em que fundamentam várias teorias e modelos de ensino, são considerados necessários para um eficaz e eficiente ensino. Decorre também desta premissa que haverá uma diminuição na aprendizagem quando um programa ou uma prática viola ou não implementa um ou mais destes princípios.

A maior parte dos modelos de ensino admite que os melhores produtos ou meios ambientes de aprendizagem estão focados em problemas e implicam quatro fases: ativação das experiências anteriores; demonstração das competências; aplicação das competências e integração dessas competências no mundo real.

Merril (1983) desenvolveu a teoria Component Display Theory (CDT) que classifica a aprendizagem em duas dimensões: conteúdo (fatos, conceitos, procedimentos e princípios) e desempenho (recordação, uso, generalidades).

Essa teoria especifica ainda quatro formas primárias de apresentação: regras (apresentação expositiva de uma generalidade); casos (apresentação expositiva de exemplos); lembrança (investigação da generalidade); prática (investigação do caso). E as seguintes formas secundárias de apresentação: pré-requisitos; objetivos; ajuda; mnemônicas; resposta.

Para este autor, a instrução será tanto mais efetiva quanto mais contemplar as formas primárias e secundárias necessárias. Uma aula completa V. 6 № 1 , Julho, 2008 
4

deverá conter sempre um objetivo a que se seguirá um processo de combinação de regras, exemplos, lembranças, prática, resposta, ajuda e mnemônicas apropriados ao assunto da disciplina e ao trabalho de aprendizagem. De fato, a teoria sugere que para um dado objetivo e cada aluno, existe uma combinação única de formas de apresentação, que resulta na mais efetiva experiência de aprendizado.

Merrill (1999) apresentou a teoria instrutiva da transação (ITT) que tem quatro objetivos básicos: a criação de uma efetiva instrução, desenvolver o aumento da eficiência da instrução, produção de simulações instrucionais e de pequenos trabalhos e fornecer a instrução adaptável.

Merrill alega que toda a estratégia instrutiva, quer algorítmica ou experimental, pode ser descrita nos termos de métodos do ITT. Ele descreve os componentes do ambiente de aprendizagem baseado no ITT como segue:

-objetivo da instrução que se destina a promover;

-a estrutura do conhecimento requerida pelo ambiente de aprendizagem;

-o motor geral da simulação que opera sobre esta estrutura de conhecimento para representar atividades e processos que ocorrem no mundo;

-a exploração da atividade de aprendizagem pelo qual o estudante interage com 0 ambiente de aprendizagem.

Wiley (2000) para desenvolver sua teoria instrucional analisou diversas teorias, sintetizou o cerne existente nelas e identificou previamente um processo ótimo.

Para este autor, num objeto de aprendizagem o conteúdo deve ser quebrado em pequenos módulos, os quais podem ser reutilizados individualmente ou combinados com outros módulos.

Assim como Gagné (1980) também Wiley (2000) sugeriu que a aprendizagem pode ser organizada numa forma hierárquica de crescente complexidade. Wiley (2000) afirma que a importância desta hierarquia consiste em permitir identificar os pré-requisitos que devem ser satisfeitos em cada um dos níveis de forma a facilitar a aprendizagem e fornecer uma base para a sequência de instrução.

Van Merriënboer (1997) apud Wiley (2000) afirmou que os componentes da habilidade cognitiva complexa podem ser recorrentes ou não recorrentes. Cada habilidade requer um formulário de informação prévia. As habilidades recorrentes necessitam de um pré-requisito, como procedimentos, regras ou conceitos. Já as habilidades não-recorrentes precisam do conhecimento de suporte, tal como modelos casuais, conceituais ou mentais.

Para este autor, a ênfase instrutiva do projeto está em encontrar problemas instrutivos e exemplos trabalhados para o modelo de interesse. Isto é realizado através da criação dos tipos de caso. Também são projetados os problemas específicos reais dentro de cada tipo de caso. Van Merriënboer (1997) apud Wiley (2000) recomenda que os tipos de caso dentro da prática do trabalho de interesse estejam arranjados em seqüências do simples para os casos mais complexos. 
5

Wiley (2000) afirma que a aprendizagem funciona melhor em um ambiente onde 0 instrutor e o aprendiz têm uma responsabilidade comum para a aprendizagem. Para ele, assim como para Bruner (1966) o instrutor deve envolver o estudante na sua própria aprendizagem.

Wagner (1994) apud Moreno e Mayer (2007) afirma que o aluno precisa interagir com o ambiente de aprendizagem para realizar uma aprendizagem significativa. Mas para estabelecer verdadeira interatividade, o aluno precisa se sentir participante da ação.

Para Moreno e Mayer (2007) a aprendizagem mais eficaz é realizada em ambientes que combinam as representações do conhecimento em verbais (palavras impressas, palavras faladas) e não-verbais (ilustrações, fotografias, vídeo e animação), utilizando a modalidade mista para as apresentações desse conhecimento (visuais e auditivas).

Estes autores afirmam que existem cinco tipos diferentes de interagir com o sistema como é apresentado no quadro abaixo:

Quadro 01- Cinco tipos de interatividade em ambientes de aprendizagem

\begin{tabular}{|c|c|c|}
\hline Tipo de interatividade & Descrição & Exemplo \\
\hline Dialogar & $\begin{array}{l}\text { Recebe perguntas e respostas } \\
\text { ou comentários. }\end{array}$ & $\begin{array}{l}\text { Procurar a ajuda de um } \\
\text { agente na tela, clicar em um } \\
\text { hiper-link para obter } \\
\text { informações adicionais. }\end{array}$ \\
\hline Controlar & $\begin{array}{l}\text { Determina ritmo e/ou de } \\
\text { ordem de apresentação. }\end{array}$ & $\begin{array}{l}\text { Usar botão de } \\
\text { pausa/prosseguir, enquanto } \\
\text { observa uma animação } \\
\text { narrada. }\end{array}$ \\
\hline Manipular & $\begin{array}{l}\text { Estabelece parâmetros de } \\
\text { uma simulação, ou faz um } \\
\text { zoom para dentro ou para } \\
\text { fora, ou move objetos ao redor } \\
\text { da tela. }\end{array}$ & $\begin{array}{l}\text { Configurar parâmetros em } \\
\text { uma simulação do jogo e } \\
\text { executar a simulação para ver } \\
\text { o que acontece. }\end{array}$ \\
\hline Pesquisar & $\begin{array}{l}\text { Encontrar novo material do } \\
\text { conteúdo, inserir uma } \\
\text { consulta, receber opções, e } \\
\text { selecionar uma opção. }\end{array}$ & $\begin{array}{l}\text { Procurar informações de uma } \\
\text { pesquisa na Internet. }\end{array}$ \\
\hline Navegar & $\begin{array}{l}\text { Mover-se para áreas de } \\
\text { diferentes conteúdos através } \\
\text { da seleção de várias fontes de } \\
\text { informação disponíveis. }\end{array}$ & $\begin{array}{l}\text { Clicar em um menu para } \\
\text { passar de uma página para } \\
\text { outra na Internet. }\end{array}$ \\
\hline
\end{tabular}

Retirado de Moreno e Mayer (2007),traduzido por M. Lucia P. Flôres

Moreno e Mayer (2007) resume cinco princípios com base empírica para a concepção de ambientes de aprendizagem interativa, junto com sua correspondente fundamentação teórica: atividade dirigidas, reflexão, feedback, ritmo, e pré-requisito.

Quadro 02 - Cinco princípios do design e a fundamentação teórica correspondente 
6

\begin{tabular}{|c|c|}
\hline Atividades dirigidas & \\
\hline $\begin{array}{l}\text { Os alunos aprendem melhor quando interagem } \\
\text { com um agente pedagógico que orientam a } \\
\text { sua atividade }\end{array}$ & $\begin{array}{l}\text { Processo cognitivo guiado - faz com que o } \\
\text { aluno empenha-se ativamente na seleção, } \\
\text { organização e integração de novas } \\
\text { informações, gerando a transformação } \\
\text { essencial. }\end{array}$ \\
\hline \multicolumn{2}{|l|}{ Reflexão } \\
\hline $\begin{array}{l}\text { Os alunos aprendem melhor quando refletem } \\
\text { sobre as respostas corretas durante o } \\
\text { processo de aprendizagem. }\end{array}$ & $\begin{array}{l}\text { A reflexão promove significado na } \\
\text { transformação essencial e geradora, } \\
\text { incentivando na organização e integração de } \\
\text { novas informações. }\end{array}$ \\
\hline \multicolumn{2}{|l|}{ Resposta (feedback) } \\
\hline $\begin{array}{l}\text { Os alunos aprendem melhor com exposição ao } \\
\text { invés da correção direta. }\end{array}$ & $\begin{array}{l}\text { As respostas explicativas fornecem aos alunos } \\
\text { um bom esquema de reparar os seus } \\
\text { equívocos. } \\
\text { Só o feedback reduz alheias transformação. }\end{array}$ \\
\hline \multicolumn{2}{|l|}{ Estimulação (ritmo) } \\
\hline $\begin{array}{l}\text { Os alunos aprendem melhor quando podem } \\
\text { controlar o ritmo da apresentação dos } \\
\text { materiais instrucionais. }\end{array}$ & $\begin{array}{l}\text { A Estimulação permite aos novos alunos } \\
\text { reduzir a exploração representacional, } \\
\text { minimizando a quantidade de informação que } \\
\text { precisa ser processada na memória de } \\
\text { trabalho em uma hora. } \\
\text { Uma solução para que haja tempo suficiente } \\
\text { para a transformação é o de quebrar a } \\
\text { animação e explicação correspondente em } \\
\text { segmentos pequenos. Desta forma, os } \\
\text { estudantes são capazes de selecionar as } \\
\text { palavras e imagens a partir de um segmento e } \\
\text { organizá-las antes de passar para o próximo } \\
\text { segmento. }\end{array}$ \\
\hline \multicolumn{2}{|l|}{ Pré-requisito } \\
\hline $\begin{array}{l}\text { Os alunos aprendem melhor quando recebem } \\
\text { pré-requisitos que fornecem ou ativam } \\
\text { relevantes conhecimentos prévios. }\end{array}$ & $\begin{array}{l}\text { Pré-requisito ajuda a orientar o aluno na } \\
\text { transformação geradora, mostrando os } \\
\text { aspectos de conhecimento prévio para integrar } \\
\text { a informação recebida. }\end{array}$ \\
\hline
\end{tabular}

Retirado de Moreno e Mayer (2007),traduzido por M. Lucia Flôres

\section{3- TIPOS DE OBJETOS DE APRENDIZAGEM}

O uso da tecnologia e a aprendizagem significativa estão interrelacionadas.

Para Moreno e Mayer (2007) os ambientes de aprendizagem interativos melhoram a maneira como as pessoas aprendem. Esses autores sugerem vários tipos de ambientes interativos descritos a seguir:

Jogos interativos e simulações: melhoram a aprendizagem baseado na Web, pois os usuários as consideram divertidas e motivadoras (Gee (2004) apud Moreno e Mayer (2007).

Agentes pedagógicos: fornecem linhas de orientação, aconselhamento, feedback, e apoio adequado às necessidades do aluno. Cassell et al.(2000) apud Moreno e Mayer (2007) argumentam que agentes podem servir como 
7

tutores que não só ajudam os alunos a aprender, mas também ajudam a encontrar estratégias para uma aprendizagem eficaz.

Materiais pedagógicos: é tendência crescente para o desenvolvimento e a utilização de materiais pedagógicos como uma estratégia de resolução de problemas. Estas ferramentas geralmente são instrucionais e apresentadas em texto ou vídeo (Beck et al. (2002) apud Moreno e Mayer (2007)).

Bibliotecas digitais: os alunos têm acesso a grandes quantidades de informações através de várias bibliotecas digitais (exemplo: o World Wide Web $(w w w))$. As bibliotecas digitais tornam-se indispensáveis para o aprendizado. No entanto, os estudos sobre a utilização das bibliotecas digitais indicam que os estudantes precisam de muita orientação (Dillon e Jobst (2005) apud Moreno e Mayer (2007)).

Wiley (2000) afirma que ao construir um objeto de aprendizagem, o construtor deve determinar se a teoria instrutiva é um modelo inédito, um modelo com variações diferentes para circunstâncias diferentes, ou um modelo independente dos componentes onde um instrutor pode escolher os métodos $e$ as estratégias para suas finalidades. Após fazer essa verificação é que o construtor vai estudar qual o tipo de objeto é mais adequado para um determinado conteúdo e que venha facilitar a aprendizagem. Em sua teoria, Wiley (2000) adotou cinco tipos diferentes de objetos de aprendizagem, todos eles com a característica de serem reusados em diferentes contextos. Esses tipos de objetos estão descritos a seguir, e aqui também é mostrado como eles devem ser projetados.

3.1-Fundamental

É um recurso digital individual. O objeto de aprendizagem do tipo fundamental deve ser projetado em função do maior número de contextos possíveis. Deve consistir em um elemento individual de um único tipo de meio. São exemplos de objeto de aprendizagem fundamental: uma imagem escaneada da imagem da Mona Lisa; um texto; uma citação.

3.2-Combinado-inédito

É caracterizado por um pequeno número de recursos digitais combinados. Os objetos de aprendizagem combinado-inéditos devem ter uma única finalidade, isto é, devem fornecer a instrução ou a prática.

Eles devem ser projetados para apresentar uma informação inteira (única) ou parte de informação autônoma, e devem ser restritos à combinação de dois a quatro elementos, pois combinar um número maior vai contra o princípio da reutilização. Possui uma lógica limitada e muitos ambientes estáticos, têm linguagem com vários comandos, que podem ser usadas para criar um item simples. O objeto combinado-inédito não pode ser usado em tantos contextos como o fundamental. São exemplos de um objeto de aprendizagem combinado inédito: um mapa (a combinação da imagem estática com etiquetas do texto) e um filme digital (a combinação do vídeo e do áudio).

3.3-Combinado-modificável

É caracterizado por um número maior de. recursos digitais combinados. Eles freqüentemente combinam a instrução e a prática - fornecendo e V. 6 № 1 , Julho, 2008 
8

relacionando combinações e objetos do tipo fundamental em ordem para criar a sequência lógica e instrutiva completa.

Esses objetos parecem ser simples de projetar, pois eles são apenas a combinação de outros objetos de aprendizagem. Entretanto, como sua finalidade é ser "instrucional," fica difícil de projetá-lo. Pois os objetos do tipo fundamental e os combinado-inéditos dificilmente podem ser arranjados em seqüência instrutiva quando eles forem projetados para atingir o maior número de contextos possíveis. Os objetos combinado-modificáveis podem ser projetados de tal maneira que eles possam ser reusáveis como um todo. Por exemplo, a história da Mona Lisa e uma exposição das qualidades artísticas dela são menos reutilizável do que somente a imagem, mas todo o objeto combinado-modificável que contem a imagem, a história e a exposição podem ser usados em muitas maneiras diferentes.

\section{4-Gerador-apresentação}

É caracterizado pela lógica e estrutura para combinar ou gerar e combinar objetos de aprendizagem de nível baixo (fundamental e combinado-inédito) para criar apresentações para o uso em instrução, em prática e em ensaios. Eles têm a reusabilidade intra-contextual elevada, isto é, eles podem ser usados repetidamente em contextos semelhantes. Mas a reusabilitade inter-contextual é relativamente baixa (o uso do conteúdo fica restrito ao contexto em que foi projetado).

Eles devem ser projetados conforme as diretrizes similares do combinadomodificável com as considerações adicionais de arquivos de dados e de lógica para criar as apresentações específicas.

3.5-Gerador-instrução

É caracterizado pela lógica e estrutura para combinar objetos de aprendizagem (fundamental e combinado-inédito) e avaliar as interações do estudante com essas combinações, criadas para suportar as estratégias do sumário instrutivo (como exemplo, lembrar e executar uma série das etapas).

Um exemplo deste tipo de objeto é um ambiente de aprendizagem baseado na teoria instrutiva da transação de Merrill (1999). Por exemplo, toda transação das interações que deve ocorrer para que um estudante consiga atingir um determinado tipo de aprendizagem.

Neste tipo de objeto as reusabilidades intra-contextual e inter-contextual são elevadas.

\section{4-DIRETRIZES PARA SELECIONAR OS TIPOS DE OBJETO DE APRENDIZAGEM}

Segundo Wiley (2000), antes de iniciar o projeto instrutivo de objetos de aprendizagem, deve-se decidir qual o tipo de objeto a se usar.

Van Merriënboer (1997) apud Wiley (2000) sugere para conteúdos altamente recorrentes os objetos do tipo gerador, já para conteúdos (heurísticos) altamente não-recorrentes os do tipo combinado. Este autor recomenda usar objetos de aprendizagem gerador sempre que possível; se não, usar objetos combinados. 
9

Wiley (2000) especifica com mais detalhes estas diretrizes, selecionando para cada conteúdo ou habilidade um tipo específico de objeto.

4.1-Cada gráfico, vídeo, áudio, textos individuais, animação, e elemento do software projetado para o uso no ambiente de aprendizagem por computador deve ser projetado como um objeto de aprendizagem fundamental, arquivado, e indexado como metadado.

4.2- Referências, a Ajuda, as informações do pré-requisito ou de suporte devem ser projetadas como gerador-instrução, fundamental ou objetos combinadoinédito, nesta ordem da preferência.

4.3- Se as habilidades forem algorítmicas, selecionar ou projetar objetos de aprendizagem gerador-instrucão para cada tipo de caso. Se as habilidades forem heurísticas projetar o modelo combinado-modificável.

4.4- Se os objetos de aprendizagem gerador-instrução forem criados previamente, os problemas específicos serão criados pelo computador. Se não, os problemas específicos devem ser projetados como objetos combinadomodificáveis, de modo que o objeto projetado previamente para a apresentação da informação e o problema sejam combinados dinamicamente nesse específico objeto.

4.5- Qualquer objeto (fundamental ou combinado-inédito) para compor um objeto de aprendizagem gerador-instrução deve ser projetado como a geradorapresentação, fundamental ou objetos combinado-inéditos, nesta ordem. Estes objetos podem ser apresentados no contexto de problemas múltiplos, de exemplos trabalhados ou de instrução.

4.6- Projetar objetos de aprendizagem combinado-modificáveis para cada modelo de trabalho. Usar objetos combinado-modificáveis para modelos do trabalho permite ao designer que:

(a) projete problemas específicos e seus componentes como os objetos de aprendizagem independentes que podem ser reusados em outros modelos do trabalho,

(b) combina e arranja na seqüência o modelo de trabalho.

4.7-Projetar objetos de aprendizagem gerador-instrução ou combinadomodificáveis para os modelos de trabalho integrado. Alguns objetos geradorapresentação, combinado-inédito e fundamental desenvolvidos previamente podem ser reusados para popularizar este objeto. Os objetos inéditos devem ser criados como objetos de aprendizagem gerador-apresentação, fundamental ou combinado-inédito.

\section{5-CONSIDERAÇÕES FINAIS}

Neste artigo mostrou-se que a aprendizagem pode ser realizada por apenas um recurso, como uma imagem, ou ainda, usando vários recursos digitais combinados.

Um objeto de aprendizagem pode ter só a instrução, ou combinar a instrução e a prática. Podem, ainda, ter uma lógica e uma estrutura para gerar e/ou combinar com outros objetos de aprendizagem, podendo ou não avaliar as interações dos estudantes com esse objeto.

V. 6 № 1, Julho, 2008 
10

Mas o construtor do objeto de aprendizagem deve se preocupar com a interação que o aluno terá com um determinado objeto para que o fim maior seja uma aprendizagem significativa, e que esse recurso venha contribuir para facilitar o processo de aprendizagem do estudante.

\section{7-REFERÊNCIAS BIBLIOGRÁFICAS}

-BRUNER, J. Toward a Theory of Instruction. Cambridge, MA: Harvard University Press, 1966.

-DAHL, O. J. \& NYGAARD, K. SIMULA - An algol based simulation language. Communications of the ACM, 9 (9), 1966. p. 671-678.

-GAGNE, R. M. Princípios essenciais da aprendizagem para o ensino. Tradução de Rute V. Ângelo. Porto Alegre: Globo, 1980.

-MARTINS, M.de L. O. O papel da usabilidade no ensino a distância mediado por computador. Dissertação de Mestrado. fl.107 Centro federal de Educação tecnológica de Minas Gerais - CEFET , 2004.

-MERRIL, M.D. Instructional design theory. Englewood Cliffs, NJ:Educational Technology Publications, 1983.

-MERRIL, M. D. Instructional transaction theory (ITT): Instructional design based on knowledge objects. In C. M. Reigeluth (Ed.), Instructional design theories and models: A new paradigm of instructional theory. (pp. 397424). Hillsdale, NJ: Lawrence Erlbaum Associates, 1999.

-MORENO, R e MAYER, R. Interactive Multimodal Learning Environments Special Issue on Interactive Learning Environments: Contemporary Issues and Trends. Disponível em:

http://www.springerlink.com/content/v5414u250220511r/fulltext.html Acessado em 29/05/2008.

-WILEY, D. A. (2000). Learning object design and sequencing theory . Unpublished doctoral dissertation, Brigham Young University. Disponível em: http://www.reusability.org/read/chapters/wiley.doc Acessado em: 22/05/2008. 